\title{
Exploration of NPTX2 in Islets of Langerhans
}

\author{
Lindsay Wald \\ University of Florida
}

Faculty mentor: Martha Campbell-Thompson, Department of Pathology

\begin{abstract}
NPTX2 (neuronal pentraxin-2) is a synaptic protein found abundantly in only two locations in a healthy human body: the brain and the pancreas, specifically islet of Langerhans cells. NPTX2's role in the brain has been a focus of study in the pathology of Parkinson's disease, as it is upregulated in PD patients. Its primary functions in the brain are to establish excitatory synapses and to recruit alpha-amino-3-hydroxy5-methyl-4-isoxazolepropionic acid (AMPA)-type glutamate receptors to said synapses. These AMPA receptors signal for the neurotransmitter, glutamate, that regulates insulin secretion. This is of pathological significance to the onset of type 1 diabetes. Type 1 diabetes is characterized by the depletion of islet $\beta$-cells in the pancreas, which are responsible for insulin secretion. Without a supply of insulin, fatal consequences will ensue. NPTX2's function in the pancreas is unstudied and extremely relevant to unraveling the complex processes that the body undergoes with the onset of this autoimmune disease. In recent mRNA studies, NPTX 2 mRNA was significantly downregulated in type 1 diabetes. To understand the underlying cause of this downregulation and its potential role in the destruction of islet $\beta$-cells, it is first necessary to localize NPTX2 in the islet cells of type 1 diabetic, auto-antibody positive, and control donors. Immunofluorescent staining indicates that NPTX2's co-expression in $\alpha$-cells is $0.41 \%$ in control, $1.85 \%$ in type 1 diabetic, and $1.11 \%$ in auto-antibody positive. Its co-expression in $\beta$-cells is $92.20 \%$ in control, $93.65 \%$ in type 1 diabetic, and $92.29 \%$ in auto-antibody positive. The data present indicates that NPTX2 is primarily co-expressed in $\beta$-cells of the islets of Langerhans with a small concentration in $\alpha$ cells.
\end{abstract}

Keywords: type 1 diabetes, NPTX2, pancreas

\section{Introduction}

Type 1 diabetes is an autoimmune disease in which the body stops producing the hormone insulin. Insulin is responsible for sequestering blood glucose into cells for energy use. Without it, glucose will build up in the bloodstream at toxic levels, which results in lethal consequences. The pancreas is made up of two cell types: exocrine and endocrine cells. The islets of Langerhans make up the primary endocrine cell type in the pancreas and are responsible for the release of insulin and glucagon, two major hormones in the body. The onset of this disease has been characteristically described as an autoimmune attack on the islet $\beta$-cells in the pancreas. The destruction of the $\beta$-cells, which produce insulin, leads to little to no insulin available for the body (Atkinson, Eisenbarth, \& Michels, 2014). The processes that induce this autoimmune response remain undiscovered in research. With recent mRNA studies, it has been identified that 
NPTX2, a synaptic protein, is significantly downregulated in islets from donor organs with type 1 diabetes (data unpublished).

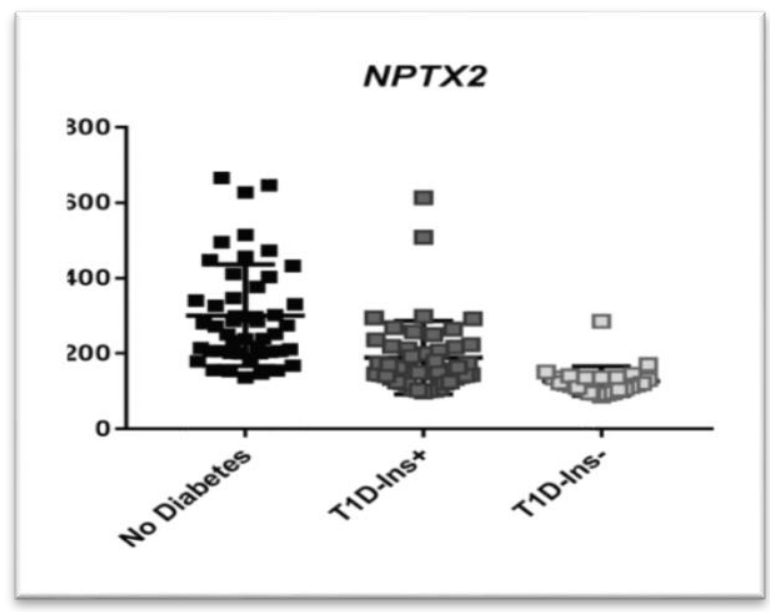

Figure 1: NPTX2 mRNA levels are observed in non-diabetic, human donors, type 1 diabetic donors with residual $\beta$ cells (T1D-Ins+), and type 1 diabetic donors without $\beta$-cells (T1D-Ins-). The graph indicates a positive correlation between the number of $\beta$-cells and the NPTX2 mRNA levels.

Investigating the processes behind the downregulation will provide information on how its downregulation affects the $\beta$-cells and methods to maintain its expression in the islet. Measures to prevent this event from occurring could increase function and proliferation of $\beta$-cells in the pancreas.

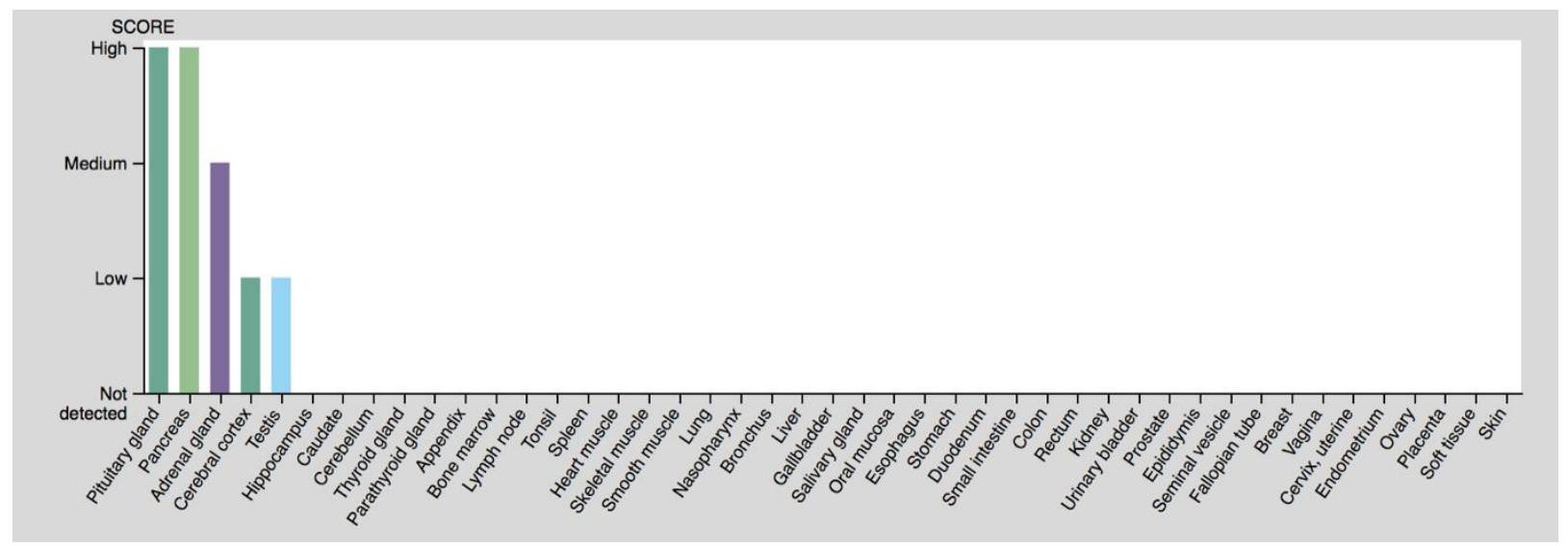

Figure 2: Bar graph displaying the relative mRNA levels of NPTX2 in normal human tissue (The Human Protein Atlas, n.d.)

NPTX2, neuronal pentraxin 2, belongs to a family of neuronal pentraxins (NPTX1, NPTXR). Pentraxins's defining feature is their ability to bind to a vast number of ligands such as 
phosphorylcholine, chromatin, DNA, immune complexes, and more. This feature suggests that this family of proteins may regulate the uptake of the cellular debris associated with immune responses and inflammation (Omeis, Hsu, \& Perin, 1995). Studies done regarding NPTX2's role in the body have been primarily from its role in the pathology of Parkinson's Disease. In the brain, it is known to bind to excitatory synapses and gathers AMPA-type-glutamate receptors at the extracellular surface of the synapses. Its carboxyl terminal allows it to bind to the $\mathrm{N}$-terminal domain of the AMPA receptors (Xiao, et al., 2017). AMPA receptors are multimeric, homologous subunits of GluR1-GluR4. These AMPA-type glutamate receptors function to regulate insulin secretion from islet $\beta$-cells (O’Brien et al., 1999). NPTX2 can also act at presynaptic terminals and induce a postsynaptic excitatory response. These capabilities of NPTX2 foster its role in preservation of synaptic plasticity (Xiao, et al., 2017). Its function in the pancreas is unknown and is of great importance given its limited prevalence in the body. It is first necessary to assess NPTX2's location in the islets of Langerhans. The use of control, autoantibody positive, and type 1 diabetic donors will determine the location of NPTX2 in the islets and whether its location changes with the onset of the disease. Current research suggests that NPTX2 will not change location with the onset of the disease, but rather show a decrease in overall localization in the islet cells. Auto-antibody positive donors are individuals who display at least one antibody against $\beta$-cells (Calderon \& Sacks, 2014). Common antibodies include GAD, insulin, IA-2, and zinc transporter 8. Those who develop these antibodies are at a much greater risk of developing type 1 diabetes (Bonifacio, 2015).

\section{Methods}

\section{Immunofluorescent staining}

$4 \mu \mathrm{m}$ thick slices of pancreas body and tail of control, auto-antibody positive, and type 1 diabetic donors were received from JDRF nPOD as paraffin slides. To remove the paraffin wax, each slide was placed in a series of tubes for 3 minutes each: xylene, xylene, 100\% ETOH, 95\% ETOH, 75\% ETOH, PBS. Afterward, $5 \mathrm{ml}$ of 20x DAKO pH 6 target solution was diluted with $50 \mathrm{ml}$ of $\mathrm{ddH}_{2} \mathrm{O}$ to achieve a $10 \%$ solution. This solution was placed in a slide container with the slides and into a water-bath for retrieval at $97^{\circ} \mathrm{C}$ for 20 minutes. Once the 20 minutes expired, the slide container was removed from the water-bath and the lid was placed half-off for another 20 minutes to allow the slides to slowly equilibrate to room temperature. The slides were removed 
from the slide container and rinsed with PBS. The slides were then blocked with $10 \%$ normal goat serum, diluted with PBS, for one hour at room temperature. Afterward, the slides are washed 3 x 5 mins with PBS and then the first primary antibody (DAKO Gt $\alpha$ Insulin, 1:100 dilution in $10 \%$ NGS) is applied and left overnight in $4^{\circ} \mathrm{C}$. The next day, the slides are washed 3 x 5 mins in PBS and a secondary antibody (Gt $\alpha$ Gp555) is applied for 1 hour at room temperature. The slides are then washed in PBS for $3 \times 5$ mins at room temperature and the second primary antibody (Sigma Gt $\alpha$ NPTX2, 1:75 dilution in 10\% NGS) is applied and left overnight at $4^{\circ} \mathrm{C}$. The following day, the slides are washed $3 \times 5$ mins in PBS at room temperature and a secondary antibody (GtaRb488) is applied and left on for 1 hour. The slides are then washed in PBS for 3 x 5 mins at room temperature and a pre-conjugated antibody (BD Biosciences Gt $\alpha$ Glucagon AF405, 1:200 in 10\% NGS) is applied and left overnight at $4^{\circ} \mathrm{C}$. The final day, the slides are washed for 3 x 5 mins in PBS and then the final antibody (Abcam Nuclear Yellow, 1:500 dilution in $\mathrm{ddH}_{2} \mathrm{O}$ ) is applied and left on for 10 minutes. The slides are washed in PBS for 3 x 5 mins and then mounted to a coverslip with Prolong without DAPI.

Table 1. Description of JDRF nPOD Cases used.

\begin{tabular}{cccccc}
\hline Case ID & Type of Case & $\begin{array}{c}\text { Age at Onset } \\
\text { (years) }\end{array}$ & $\begin{array}{c}\text { Diabetes duration } \\
\text { (years) }\end{array}$ & $\begin{array}{c}\text { Age of demise } \\
\text { (years) }\end{array}$ & $\begin{array}{c}\text { Autoantibody } \\
\text { Results }\end{array}$ \\
\hline 6384 & Control & - & - & 17 & Negative \\
6368 & Control & - & - & 38.3 & Negative \\
6197 & Aab+ & - & - & 22 & GADA+ IA-2A+ \\
6167 & Aab+ & - & - & 37 & IA-2A+ZnT8A+ \\
6310 & Aab+ & - & - & 28 & GADA+ \\
6424 & Aab+ & - & - & 17.65 & GADA+ mIAA+ \\
6429 & Aab+ & - & 2 & 22.1 & GADA+ mIAA+ \\
6371 & T1D & 10.5 & 2 & 12.5 & GADA+ IA-2A+ \\
6396 & T1D & 15.1 & 0 & 17.1 & mIAA+ ZnT8A+ \\
6362 & T1D & 24.9 & 8 & 24.9 & Negative \\
6046 & T1D & 10.8 & & 18.8 & GADA+ \\
& & & & & Zn-2A+
\end{tabular}

Note. Pancreatic tissue used is from JDRF. This graph provides the profiles of the cases used in the experiment. All data was gathered from JDRF nPOD (JDRF nPOD Online Pathology Information, n.d.). 


\section{Image Analysis}

Images were taken at 20x magnification on a Zeiss 710 confocal microscope. A script was generated using ImageJ to calculate the Mander's correlation coefficient of NPTX2 co-localized with insulin and NPTX2 co-localized with glucagon based on previous reported methods (Canzano, et al., 2018). Using Mander's overlap coefficient equation, a value is obtained that identifies how much overlap of location exists between specific protein(s) and/or hormone(s) within an islet cell.

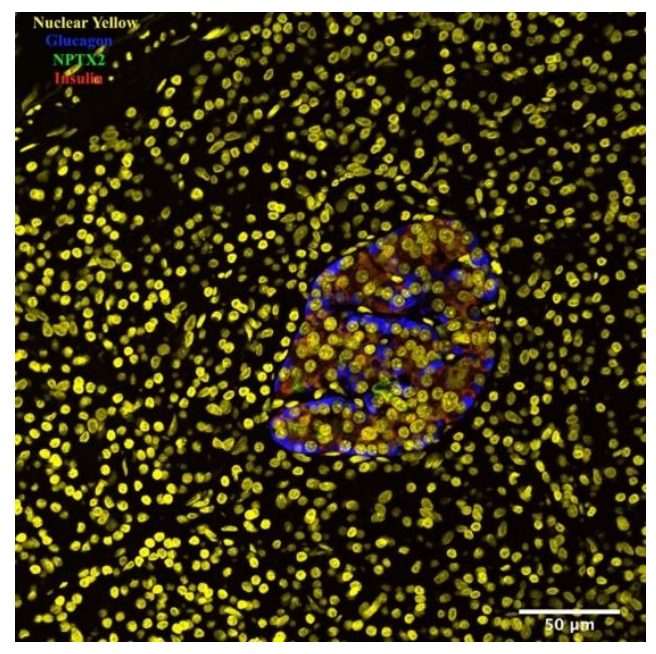

Figure 3: Original image taken on Zeiss 710 Confocal Microscope.

The arrows below indicate the creation of a threshold for image analysis for each protein/hormone stain on the slide.
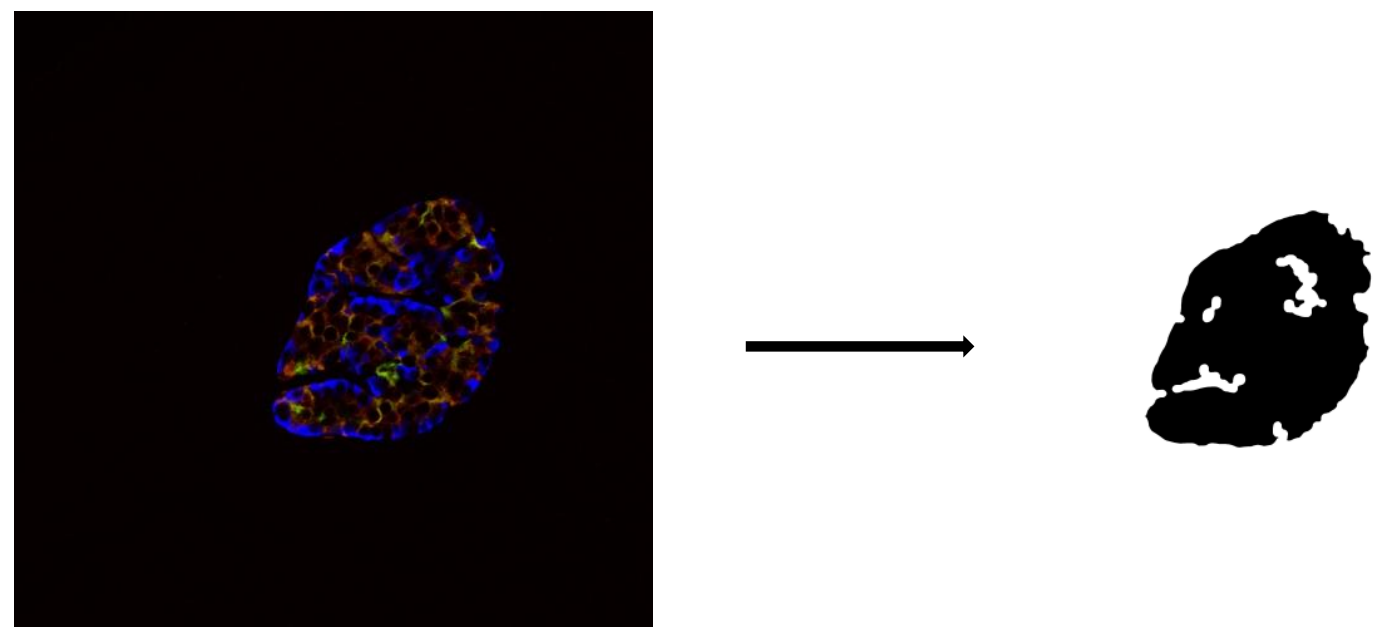

Figure 4: Thresholding of the whole islet. 


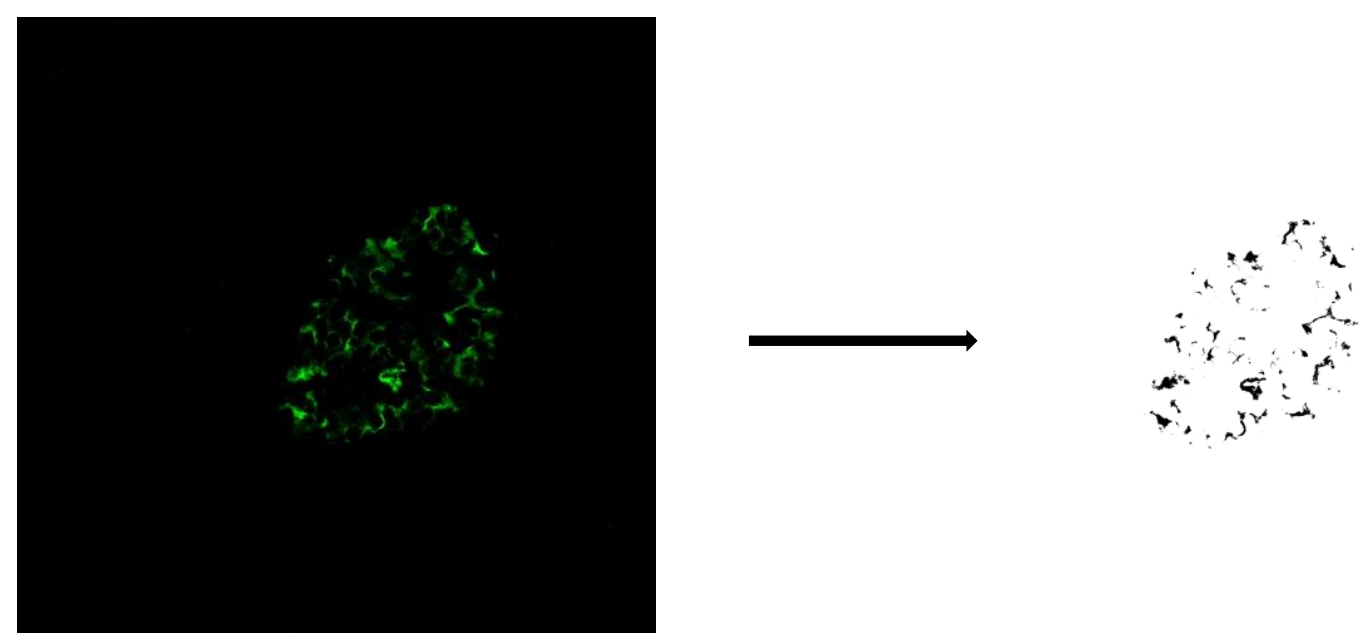

Figure 5: Thresholding of NPTX2 in the islet.
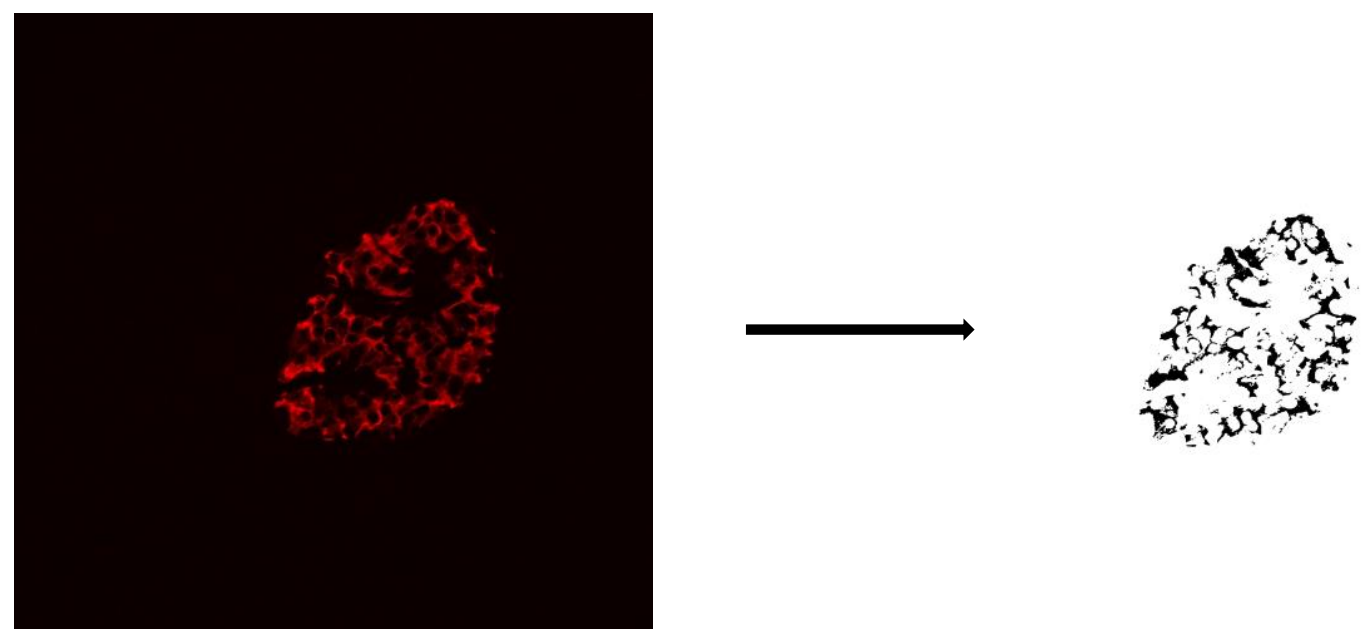

Figure 6: Thresholding of insulin in the islet.
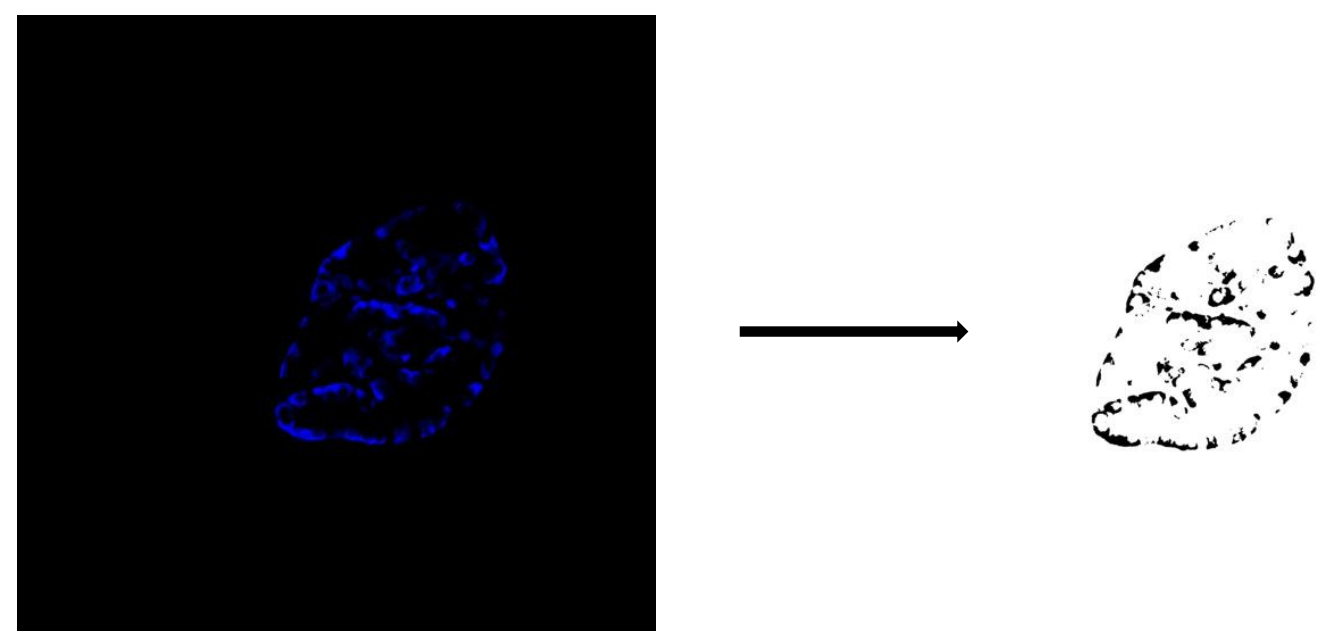

Figure 7: Thresholding of glucagon in the islet.

After thresholding, the script co-localized NPTX2 over insulin and NPTX2 over glucagon. 


\section{Results}

NPTX2 co-localization with insulin in control donors is $92.20 \%$, auto-antibody donors display $92.29 \%$ co-localization, and type 1 diabetic donors display $93.65 \%$ co-localization. An ANOVA test indicated that the differences in NPTX2 co-localization with insulin are not significant $(\mathrm{p}<.05)$. NPTX2 co-localization with glucagon in control donors is $0.41 \%$, autoantibody donors display $1.11 \%$ co-localization, and type 1 diabetic donors display $1.85 \%$ colocalization. An ANOVA test indicated statistically significant results between the three donor types $(\mathrm{p}<.05)$. Figure 8 and 9 provide a graphical model of the results.

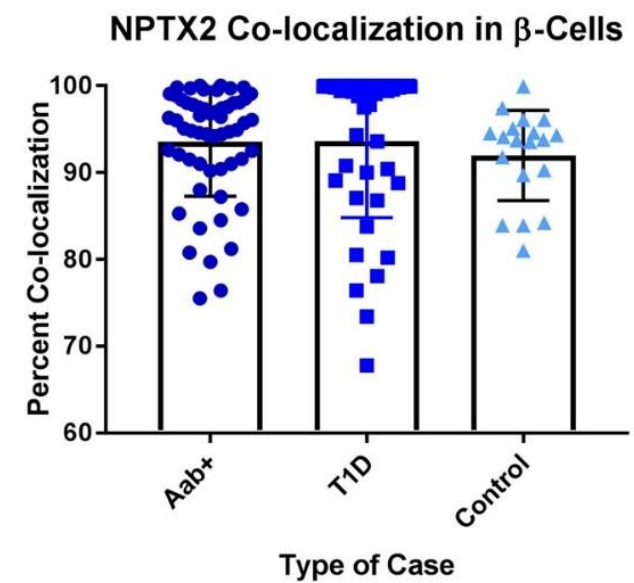

Figure 8: Percent co-localization of NPTX2 in $\beta$-cells (insulin) in autoantibody-positive, type 1 diabetic, and control donors.

NPTX2 Co-localization in $\alpha$-Cells

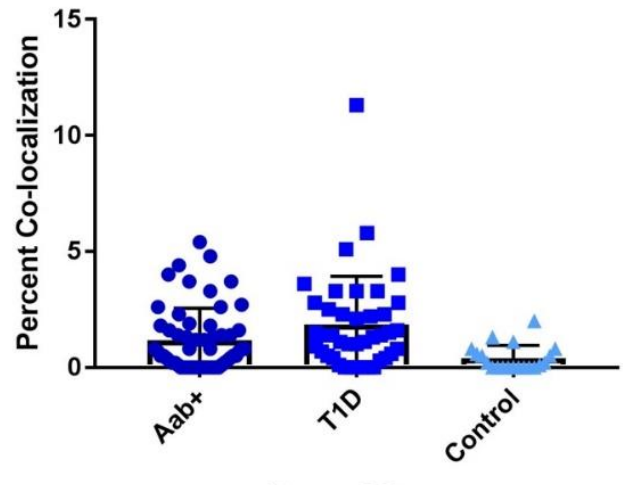

Type of Case

Figure 9: Percent co-localization of NPTX2 in $\alpha$-cells (glucagon) in autoantibody-positive, type 1 diabetic, and control donors. 


\section{Discussion}

The data indicates that NPTX2 is primarily located in $\beta$-cells. This is because of the NPTX2's role in clustering AMPA receptors that signal glutamate, a regulator of insulin secretion. NPTX2 appears to continue to co-localize its expression with insulin to the same extent in all three donor types. The destruction of $\beta$-cells with the onset of type 1 diabetes may contribute to the downregulation of NPTX2 since this protein is primarily expressed in $\beta$-cells. A small subset of NPTX2 is localized in $\alpha$-cells. NPTX2 may be produced in a small subset of $\alpha$ cells for insulin regulatory processes. The localization of NPTX2 in $\alpha$-cells is greatest in type 1 diabetic donors and smallest in control donors. Compensation for the destruction of $\beta$-cells may result in a shift in where NPTX2 protein is located. More investigation will be required to confirm these theories. Further studies regarding if there is a toxicity to NPTX2's excitatory abilities will elucidate its role in the onset of this pathology. In addition, cell and other species models can provide a comparison to the human function.

\section{References}

Atkinson, M.A., Eisenbarth, G.S., Michels, A.W. (2014). Type 1 diabetes. Lancet, 383, 69-82.

Bonifacio, E. (2015). Predicting Type 1 Diabetes Using Biomarkers. Diabetes Care, 38(6), 989996. https://doi.org/10.2337/dc15-0101

Calderon, B., Sacks, D.B. (2014). Islet Autoantibodies and Type 1 Diabetes: Does the Evidence Support Screening?. Clinical Chemistry, 60(3), 38-440. https://doi.org/10.1373/clinchem.2013.212381

Canzano, J.S., Nasif, L.H., Butterworth, E.A., Fu, D.A, Atkinson, M.A., Campbell-Thompson, M. (2018). Islet Microvasculature Alterations with Loss of Beta-cells in Patients with Type 1 Diabetes. J Histochemistry and Cytochemistry, 67(1), 41-52. https://doi.org/10.1369/0022155418778546

JDRF nPOD Online Pathology Information. (n.d.). Retrieved from https://www.jdrfnpod.org/forinvestigators/online-pathology-information/

O'Brien, R.J., Xu, D., Petralia, R.S., Steward, O., Huganir, R.L., Worley, P. (1999). Synaptic clustering of AMPA receptors by the extracellular immediate-early gene product Narp. Neuron, 23(2), 309-323. 
Omeis, I.A., Hsu, Y.C., Perin, M.S. (1995). Mouse and human neuronal pentraxin 1 (NPTX1): conservation, genomic structure, and chromosomal localization. Genomics, 28(2), 220. https://doi.org/10.1006/geno.1996.0503

Tissue expression of NPTX2 (n.d.). Retrieved from https://www.proteinatlas.org./ENSG00000106236-NPTX2/tissue

Xiao, M.F., Xu, D., Craig, M.T., Pelkey, K.A., Chien, C.C., Shi, Y.,...Worley, P.F. (2017). NPTX2 and cognitive dysfunction in Alzheimer's disease. eLife, 6, e23798. https://doi.org/10.7554/eLife.23798 